\title{
Metastatic gastric adenocarcinoma in a pregnant woman
}

\author{
Raikan Büyükavcı, Hakan Apaydın, Semra Aktürk \\ Department of Physical Medicine and Rehabilitation, Inönü University School of Medicine, Malatya, Turkey
}

DOI: $10.18621 /$ eurj.368205

\begin{abstract}
Gastric cancers occur in only $0.025 \%$ to $0.1 \%$ of all pregnancies. We present a pregnant woman with gastric adenocarcinoma associated with bone metastasis. She is 36-year-old (gravida:4, para:0) 16-week pregnancy, complaining of nausea, vomiting, weight loss $(\sim 15 \mathrm{~kg})$ and low back pain. Although the patient considered the condition to be related with pregnancy and underestimated its importance. She had high erythrocyte sedimentation rate $(107 \mathrm{~mm} / \mathrm{h})$ and anemia (hemoglobin $=9.4 \mathrm{gr} / \mathrm{dl})$. Lumbar magnetic resonance imaging showed L4 vertebrae malign compression fracture and diffuse bone metastasis. Because of the patient's melena, esophagogastroduodenoscopy was performed and biopsy result was reported as stomach adenocarcinoma. It is important to evaluate the symptoms of low back pain and pregnancy related symptoms carefully and do not cause delays in diagnosis due to overlapping symptoms.
\end{abstract}

Keywords: pregnancy, lower back pain, stomach neoplasms

Received: December 18, 2017; Accepted: January 19, 2018; Published Online: April 17, 2018

$\mathbf{L}$ ow back pain during pregnancy is a common symptom. About half of pregnancies may have complain of low back pain. There are many reasons for the etiology, such as postural changes caused by fetal growth, hormonal factors, nucleus pulpous, tumors and infections [1]. The most important cause of low back pain in pregnancy is considered to be postural changes. With the progress of pregnancy, the center of gravity slides forward and the load on the spine tends to increase [2]. The second important cause is laxity in the joints due to increased levels of the hormone relaxing during pregnancy [3, 4]. Rarely, low back pain due to primary or secondary tumors can be seen in pregnancies. Gastric cancer is occurring in only $0.025 \%$ to $0.1 \%$ of all pregnancies. Diagnosis is often difficult due to symptoms such as sickness, vomiting, or abdominal disturbance that often disappears during pregnancy [5]. In this case report, we present a case of gastric adenocarcinoma diagnosed in a pregnant patient with low back pain secondary to lumbar bone metastasis.

\section{CASE PRESENTATION}

A 36-year-old (gravida:4, para:0) 16-week pregnant woman applied to our clinic for low back pain and pain in both legs. She stated that low back pain started from the time of detection of her pregnancy, gradually increased and spreading to her right leg. She stated that her pain was increasing with the movement and sitting for a long time. Right leg pain was also accompanied by numbness and tingling. At the same time, there were some complaints such as nausea, vomiting, loss of appetite and weight loss of about $15 \mathrm{~kg}$ from the beginning of her pregnancy.

Address for correspondence: Raikan Büyükavcl, MD., Assistant Professor, İnönü University School of Medicine, Department of Physical Medicine and Rehabilitation, Malatya, Turkey

E-mail: rsoydemir@yahoo.com,Tel:+90 422 3410660,Fax:+90 4223412708 


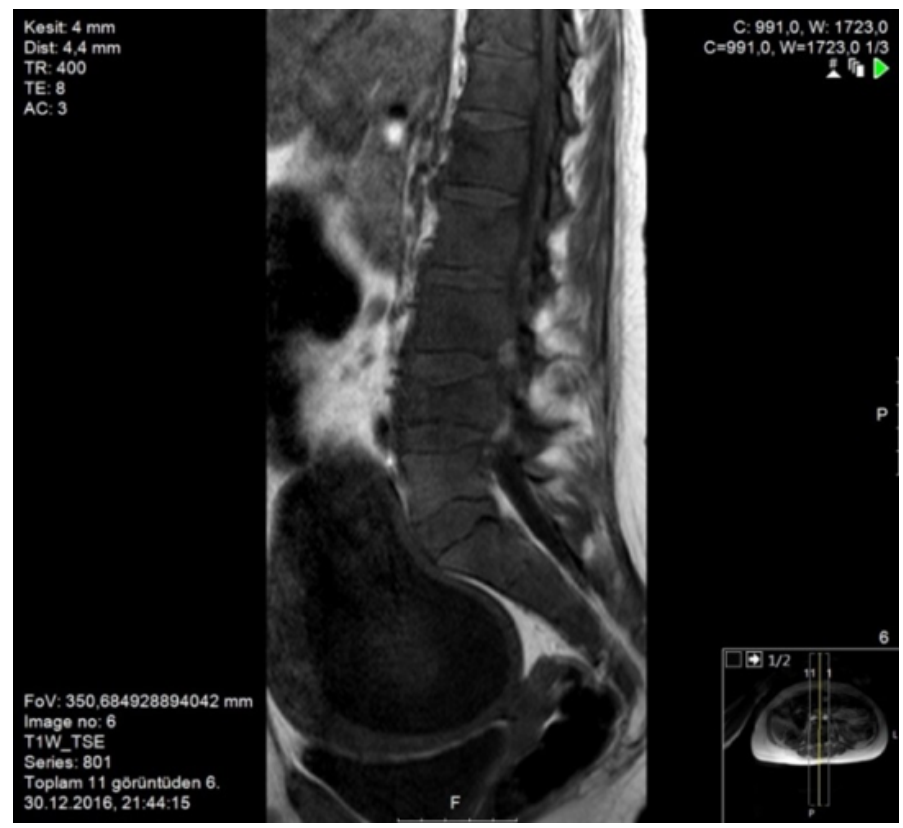

Figure 1. Lumbar MRI on T1-weighted image: L4 vertebra compression.

There was no fever or no night sweats. Her past medical and family histories were unremarkable. She went to different doctors (a neurosurgeon, a physiatrist and orthopedist) before applying to our clinic and she had no blood result with her. On physical examination, she looked pale on inspection; palpation to lumbar spinous processes and paravertebral muscles was sensitive. Lumbar region joint movements were painful and limited, especially in flexion. Lasegue test, to detect whether the low back pain has an underlying herniated disk was bilateral negative. There was a short-distance antalgic gait supported by two people. The lumbar pain level measured by visual analog scale (VAS): 10. In laboratory investigation, erythrocyte sedimentation rate was $107 \mathrm{~mm} / \mathrm{h}, \mathrm{C}$-reactive protein was $10.6 \mathrm{mg} / \mathrm{dl}$, procalcitonin was $0.101 \mathrm{ng} / \mathrm{ml}$, white blood cell account was $10.000 / \mathrm{mm} 3$, hemoglobin was $9.4 \mathrm{gr} / \mathrm{dl}$ and Brucella agglutination tests were negative. Lumbar magnetic resonance imaging (MRI) findings showed signal loss due to degeneration in L45 disc, marked heterogeneous bone marrow signal pattern in vertebral discs, marked heterogeneity in discs. The L4 vertebrae showed a height loss of less than $50 \%$, a compression fracture on $\mathrm{T} 1$-weighted image (Figure 1).

Because of the patient's melena, esophagogastroduodenoscopy was performed in the

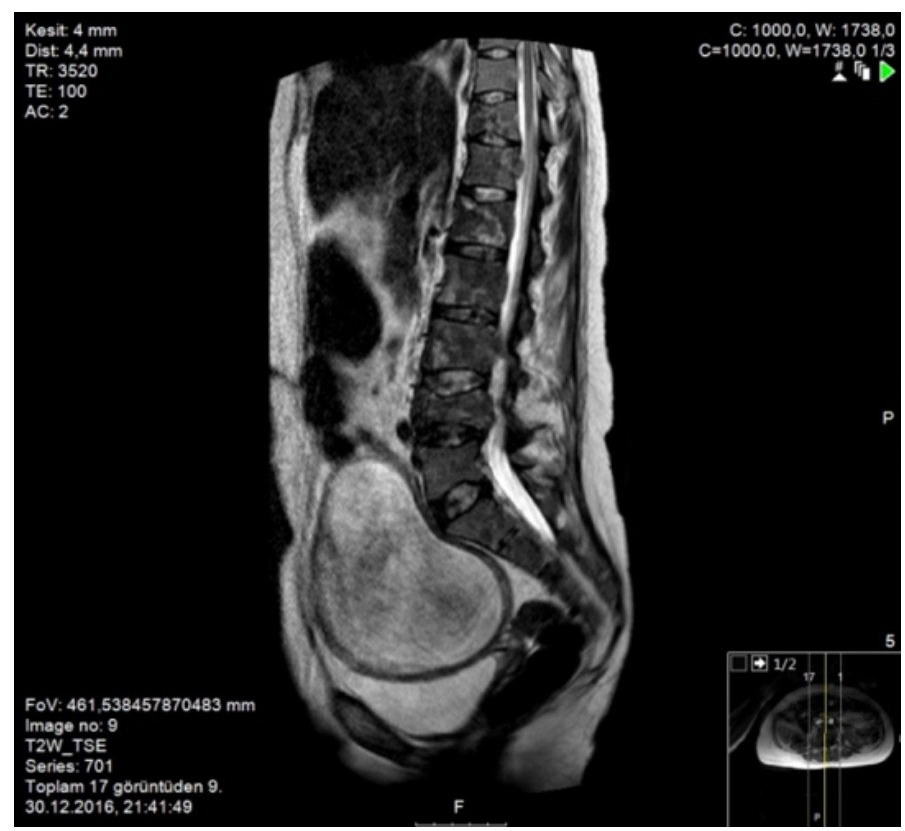

Figure 2. Lumbar MRI on T2-weighted images: L4 vertebra malignant compression fracture and diffuse bone metastasis.

gastroenterology clinic. Biopsy result was reported as stomach adenocarcinoma. In view of further examination and treatment, a lumbosacral MRI with contrast agent was obtained with the opinion of an obstetric specialist. The MRI result was evaluated as L4 vertebrae malignant compression fracture and diffuse bone metastasis on T2-weighted image (Figure 2).

Therapeutic abortus was performed with the approval of the patient and relatives. Chemotherapy and radiotherapy treatments were initiated by the oncology clinic. About one month later, the patient died due to sudden cardiac arrest.

\section{DISCUSSION}

It is known that $20-90 \%$ of pregnancies experience low back, back and hip pain, because of the laxity of ligaments and postural changes. The biomechanical load is often the cause of such pains. However, these findings are mostly confronted during the second trimester of pregnancy. Also, gestational age is found to be a risk factor for low back pain [6]. The present case was a middle-aged pregnant who applied during the 2nd trimester with low back pain, and was not different from the other cases reported. However, 
accompanying weight loss required further investigation with pathological evaluation and laboratory tests.

The incidence of cancer during pregnancy is approximately $0.1 \%$ [7], and of that gastric cancer is lower at 0.026 to $0.1 \%$ of all pregnancies [8]. In the literature, malignancies with vertebral metastases during pregnancy are mostly detected as gestational choriocarcinoma. These cases are mostly diagnosed with vertebral metastasis, after detection of a primary lesion during or after pregnancy $[9,10]$.

Pacheco et al. [5] reported 3 cases of gastrointestinal malignancy detected during pregnancy. All patients presented with epigastric pain and weight loss. A biopsy was performed by gastroendoscopy, and the cases were diagnosed as gastric cancer. The authors emphasized that gastric carcinomas are rarely seen in pregnancy, that pregnancy masks the findings of this disease, but early diagnosis improves treatment and survival, which is why it is very important to identify the disease.

Chen et al. [11] reported a case of gastric adenocarcinoma that metastasized to the placenta. In the patient who received emergency cesarean section due to 34-week preeclampsia, chylous acid was detected during surgery and it was reported as primer gastric adenocarcinoma metastasis to the intervillous space and placenta. The patient was in the 2nd trimester of pregnancy and had complaints of constant vomiting and epigastric pain. The authors noted that early gastric cancer diagnosis is delayed due to pregnancy-induced gastrointestinal problems.

Gastric cancer in pregnancy has poor prognosis [12]. Therefore, pregnancy-associated gastric cancer is extremely rare, and in many cases, it is diagnosed at an advanced stage because the symptoms during pregnancy are generally overlooked [5]. Additionally, the diagnosis of gastric cancer is often delayed in pregnant women because it is difficult to distinguish between symptoms of gastric cancer and common pregnancy-induced symptoms, such as the hyperemesis and pressure resulting from the enlargement of the uterus. Furthermore, the physician and patient are often hesitant to conduct diagnostic examinations during pregnancy $[13,14]$. One- and 2year survival rates are $18.0 \%$ and $15.1 \%$, respectively. When gastric cancer is diagnosed prior to 22 weeks of gestation, the patient should be treated after termination of the pregnancy by abortion [8].

\section{CONCLUSION}

In conclusion, our case is a rare case in the literature of gastric adenocarcinoma presenting with bone metastasis detected in the second trimester of pregnancy. Pregnancy-associated gastric cancers are extremely rare, especially with bone metastasis. It is important to carefully evaluate the symptoms of low back pain and pregnancy-related nausea, vomiting, loss of appetite and weight loss symptoms that are common in pregnancy. The accurate diagnosis due to these overlapping symptoms should not be delayed.

\section{Author Contributions}

RB: corresponding author, writing manuscript, HA: collect patient's data, and SA: edit manuscript

\section{Informed Consent}

Written informed consent was obtained from the patient for the publication of this case report.

\section{Conflict of Interest}

The authors declared that there are no potential conflicts of interest with respect to the research, authorship, and/or publication of this article.

\section{REFERENCES}

[1] Franklin ME, Conner-Kerr T. An analysis of posture and back pain in the first and third trimesters of pregnancy. J Orthop Sports Phys Ther 1998;28:133-8.

[2] Ritchie JR. Orthopedic considerations during pregnancy. Clin Obstet Gynecol 2003;46:456-66.

[3] Kristiansson P, Svardsudd K, Von Schoultz B. Serum relaxin, symphyseal pain, and back pain during pregnancy. Am J Obstet Gynecol 1996;175:1342-7.

[4] Chan YL, Lam WW, Lau TK. Back pain in pregnancy: magnetic resonance imaging correlation. Clin Radiol 2002;57:1109-12.

[5] Pacheco S, Norero E, Canales C, Martínez JM, Herrera ME, Muñoz $\mathrm{C}$, et al. The rare and challenging presentation of gastric cancer during pregnancy: a report of three cases. J Gastric Cancer 2016;16:271-6.

[6] Carvalho MECC, Lima LC, de Lira Terceiro CA, Pinto DRL, Silva MN, Cozer GA, et al. Low back pain during pregnancy. Rev Bras Anestesiol 2017;67:266-70.

[7] Cardonick E, Lacobucci A. Use of chemotherapy during human pregnancy. Lancet Oncol 2004;5:283-91.

[8] Nishie H, Mizushima T, Suzuki Y, Fukusada S, Inoue T, Kachi K, et 
al. Chemotherapy treatment of a pregnant woman with progressive gastric cancer. Intern Med. 2015;54:1207-12.

[9] Ishiguro T, Serikawa T, Yahata T, Enomoto T. Gestational choriocarcinoma: rare spinal metastasis during a viable pregnancy. $\mathrm{J}$ Obstet Gynaecol Res 2017;43:421-24.

[10] Naito Y, Akeda K, Kasai Y, Matsumine A, Tabata T, Nagao K, et al. Lumbar metastasis of choriocarcinoma. Spine (Phila Pa 1976) 2009;34:538-43.

[11] Chen Y, Li Y, Wang H, Lu J, Jin M, Zhang Z. Maternal gastric carcinoma with metastasis to the placenta: a case report. Oncol Lett
2014;8:2509-10.

[12] Cift T, Aydogan B, Akbaș M, Aydın B, Demirkiran F, Bakkaloglu $\mathrm{DV}$, et al. Case report: gastric carcinoma diagnosed at the second trimester of pregnancy. Case Rep Obstet Gynecol 2011;2011:532854.

[13] Fujino T, Nakamura M, Sato T. [Pregnancy and gastric cancer]. Shusanki Igaku (Perinatal Medicine) 2002;32:1049-55. [Article in Japanese]

[14] Jaspers VK, Gillessen A, Quakernack K. Gastric cancer in pregnancy: do pregnancy, age or female sex alter the prognosis? Case report and review. Eur J Obstet Gynecol Reprod Biol 1999;87:13-22. 